\title{
Un secolo di nursing nefrologico: Seconda parte: II consolidamento del ruolo e le sfide della modernità (1960-1990)
}

Giornale di Tecniche Nefrologiche e Dialitiche 2018, Vol. 30(I) I2-23

(C) The Author(s) 2018

Reprints and permissions:

sagepub.co.uk/journalsPermissions.nav

DOI: | 0.1 I77/03949362 |876082 I

journals.sagepub.com/home/gtn

\section{(SAGE}

\section{Sandro Geatti}

\begin{abstract}
The aim of this study is to describe the main stages in the development of nephrology nursing in its century-long history as evidenced by the existing documentation in Europe and the USA. Two key periods will be highlighted. The first, preceding the introduction of renal replacement therapy by dialysis, is distinguished by a professional attitude oriented towards preserving residual renal function and preventing complications. The second, concurrent with the introduction of dialysis, is distinguished by the need to broaden professional skills from a clinical, technical and educational point of view. The activity of eminent nurses marked these two stages. While growing, nephrology nursing changed its professional goals and adapted them to scientific progress in the broadest sense, but also to the central position of the individual in the healthcare process, the quality of outcomes, and the rational use of resources. This paper deals with a professional history that makes the nephrology nurse a unique figure in the nursing world.
\end{abstract}

\section{Keywords}

Dialyzers, kidney disease, nephrology nursing history, uremia

\section{0, soglia di una nuova epoca}

Come detto nella conclusione della prima parte, la storia della dialisi subì una svolta epocale nel marzo 1960. Fino ad allora tutti i tentativi di mantenere in efficienza un accesso vascolare per lungo tempo erano falliti, ma quasi 17 anni dopo il primo impiego del rene artificiale, Scribner (figura 1 e figura 5) e la sua équipe di Seattle diedero inizio al primo programma di trattamento di dialisi a pazienti affetti da IRC. Essi incanularono a permanenza con uno shunt esterno (figura 2) di teflon una vena ed un'arteria contigue dell'avambraccio eliminando il problema dei ripetuti cateterismi e nel contempo introdussero un approccio più nuovo e sofisticato di tecnica dialitica, usando un dializzatore a bassa resistenza realizzato da Kiil (vedi oltre) ed un sistema di preparazione del liquido di dialisi più pratico e sicuro. Crearono così le più importanti premesse per la dialisi periodica. Il primo paziente posto in trattamento fu Clyde Shields, un macchinista di 39 anni, mantenuto in vita fino al 1971 ed il cui decesso sopraggiunse a causa di un infarto miocardico.

Con una suggestiva rievocazione Scribner ricorda di essersi svegliato spontaneamente alle quattro di una mattina di quel marzo 1960 con l'insopportabile pensiero della morte imminente di Shields e di come l'insonnia facesse nascere in lui l'idea di una possibile soluzione. Egli immaginò, raffigurandolo nella sua mente uno shunt arterovenoso a forma di U tra la vena e l'arteria del polso, con una valvola che permettesse il collegamento al rene artificiale. ${ }^{1}$

La comunità scientifica fu informata dei primi risultati di Scribner in occasione del $1^{\circ}$ Congresso Internazionale di Nefrologia tenutosi a Evian, in Francia nel settembre di quell'anno. ${ }^{2}$

La fistola arterovenosa ideata da Cimino e Brescia nel $1966^{3}$ rese meno problematico il mantenimento in funzione dell'accesso vascolare perchè veniva eliminata la necessità di una protesi esterna. Il by-pass, pur modificato e migliorato

\section{Corrispondenza:}

Sandro Geatti, Dipartimento di Salute Mentale, AULSS2 Veneto,

Conegliano (TV).

E-mail: sgeatti@gmail.com 


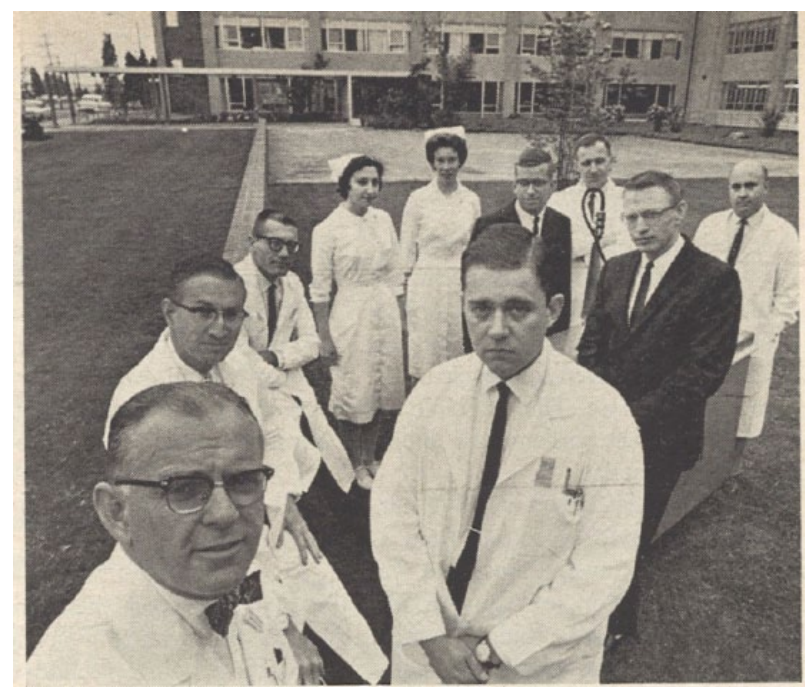

Figura I. Belding Scribner con il suo team di Seattle, Life Magazine, 1962 (Arch. Bellco).

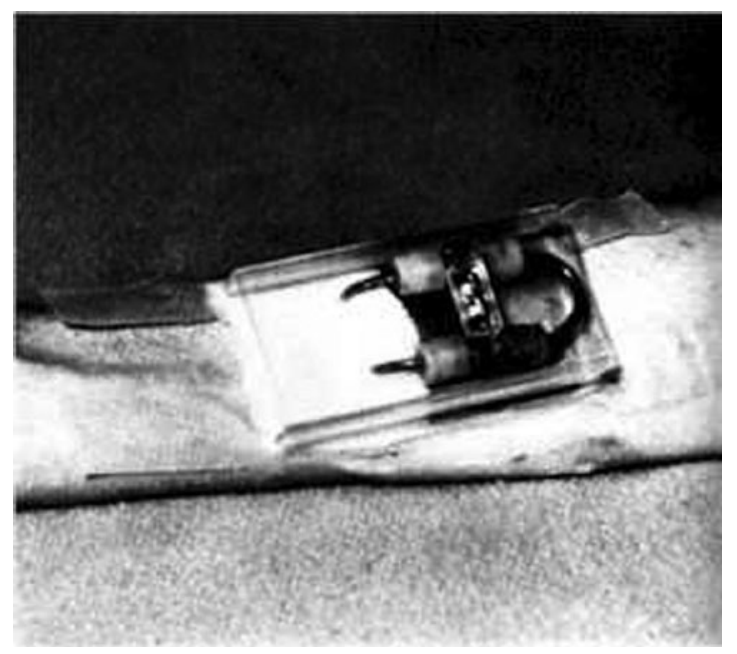

Figura 2. II primo by-pass arterovenoso esterno realizzato da Scribner nel 1960 (Arch. Bellco).

(figura 3), convisse per un altro ventennio con la fistola arterovenosa e poi fu progressivamente abbandonato.

\section{Problemi e controversie}

Nonostante il programma di dialisi consentisse di mantenere in vita un paziente affetto da insufficienza renale cronica, esso fu per qualche tempo tenuto segreto per timore di pressioni dovute ad aspettative eccessive nei pazienti, poiché si ignoravano completamente gli effetti della dialisi a lungo termine. Scribner con molta discrezione ne discusse dapprima con $\mathrm{i}$ suoi colleghi al Congresso dell'American Society for Artificial Internal Organs (ASAIO) a Chicago, e alla chiusura di quell'incontro istruì altri nove nefrologi sulle modalità di inserzione dello shunt, affinché anche essi potessero intraprendere nei loro ospedali esperienze analoghe alla sua. ${ }^{1}$

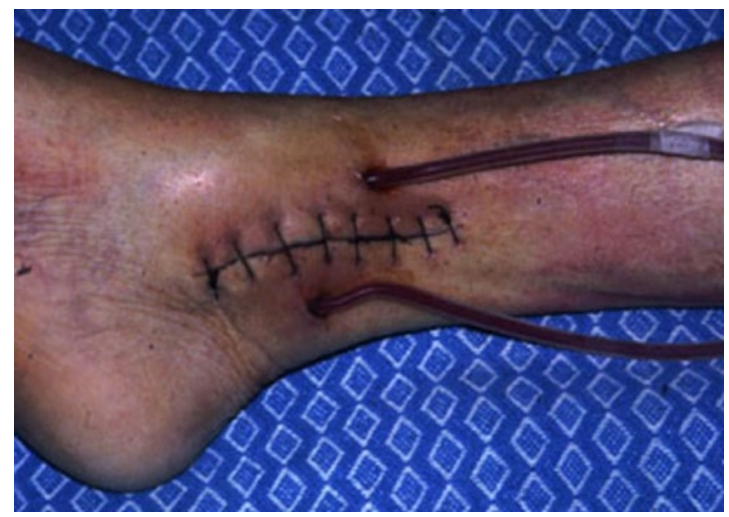

Figura 3. By-pass $A V$ con protesi esterna più evoluta, in silastic, inserita nell'arto inferiore (Arch. Bellco).

Ma le prime laceranti questioni etiche non si fecero attendere. A Seattle erano in funzione solamente tre reni artificiali per nove pazienti. Come ci si doveva comportare all'esaurimento della disponibilità? Quali criteri usare quando due o più pazienti sarebbero stati in competizione per un solo posto dialisi? E' chiaro che la scarsità di apparecchi non consentiva di assicurare la sopravvivenza a tutte le persone affette da insufficienza renale terminale (ancora nel 1967 solo il 10\% dei pazienti degli Stati Uniti poteva accedere alla dialisi ${ }^{4}$ ). Ed ancora: dove si dovevano trovare le risorse finanziarie necessarie per assicurare i futuri sviluppi della nuova tecnologia?

Il gruppo di Seattle stabilì di costituituire un comitato di selezione composto da medici e da persone di tutti i ceti sociali. ${ }^{5}$ Ciò fece scoppiare violente polemiche, tanto che Scribner una ventina di anni più tardi, sentiva ancora la necessità di doversi difendere dagli attacchi subiti: «L'enormità di questo impatto si cominciò a manifestare nel 1962, quando la giornalista Shana Alexander venne a Seattle per fare una storia del rene artificiale. Ricorderò sempre il mio sconforto nel vedere come lei non volesse sapere niente dei pazienti cui era stata salvata la vita. Lei voleva trovare tutti i dati riguardanti "il comitato della vita e della morte". Come risultato apparve su Life Magazine il suo articolo sul "comitato della vita e della morte di Seattle" (figura 4) ed iniziarono discussioni e controversie che persistono tuttora». ${ }^{6}$

Ma l'articolo della Alexander "They decide who lives, who dies» ebbe il merito di attirare l'attenzione dell'opinione pubblica su un problema che poteva essere risolto solo con il massiccio utilizzo di fondi pubblici. ${ }^{7}$

Nettamente contrari alla istituzione dei comitati etici si dichiararono i nefrologi europei, che applicarono quasi sempre il principio del first come, first served, rifiutando ogni considerazione di opportunità che non fosse di tipo clinic. ${ }^{8,9}$

A tamponare parzialmente la situazione contribuirono le meno costose pratiche della dialisi self service, introdotta in Europa da Shaldon nel $1963^{10}$ e della dialisi domicilare, iniziata dall'attivo team di Boston l'anno dopo. $^{11}$ 


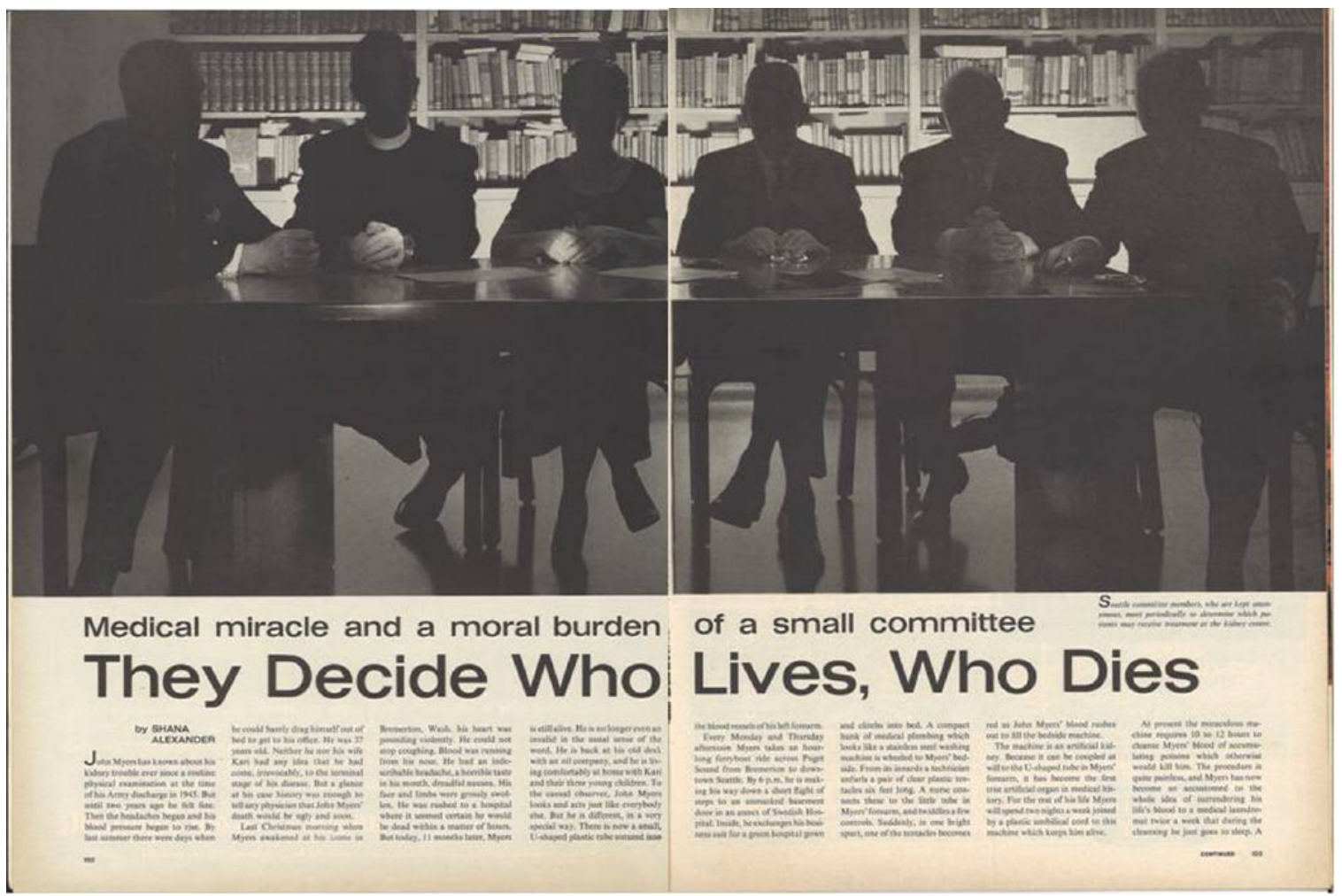

Figura 4. «They decide who lives, who dies» - Life Magazine, 1962 (Arch. Bellco).

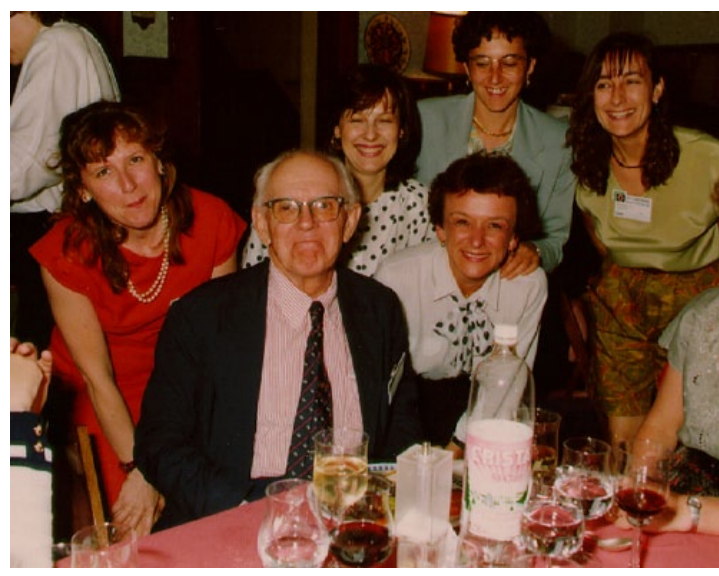

Figura 5. Il prof. Scribner al ricevimento del Congresso Europeo Edtna Erca, Bruxelles, 1996 con alcuni membri del Comitato Esecutivo Nazionale. Da sinistra: Marisa Pegoraro, Scribner, Maria Pia Zito, Gabriella Cerruti, Valentina Paris (chinata) e Margherita Rivetti (a cura dell'autore).

La comunità scientifica non fu unanime nel comprendere la reale portata della dialisi e molti dei suoi membri che pure in seguito avrebbero contribuito a perfezionarla, agli inizi degli anni sessanta si rifiutavano di pensare che la dialisi potesse venire praticata per lunghi tempi.

Racconta Alwall che ad Evian, durante il citato $1^{\circ}$ Congresso Internazionale di Nefrologia: "i lavori sul trapianto renale attrassero circa il $100 \%$ dei partecipanti al congresso. Tuttavia, solo John Merril ed il suo gruppo poterono riferire dei successi ottenuti in soli otto di nove casi di trapianto tra fratelli gemelli. Belding Scribner riferì sui quattro pazienti del suo programma mantenuti in vita da sei mesi mediante il rene artificiale ed il nuovo shunt arterovenoso percutaneo. La sua audience ammontava a 12 ascoltatori - circa 1.2\% dei partecipanti al congress". ${ }^{12}$

Anche da noi, alcune previsioni di noti nefrologi furono completamente sovvertite. ${ }^{13}$ L'assistenza sanitaria in Italia era ancora affidata al sistema mutualistico - il SSN fu istituito nel 1978 - e gli enti assistenziali tardavano a riconoscere e quindi a sostenere i costi del trattamento dialitico. Così come negli USA l'articolo della Alexander su Life, da noi fu una trasmissione televisiva a far conoscere la dialisi al grande pubblico. Il prof. Giuseppe D'Amico, primario della Divisione di Nefrologia dell'Ospedale S. Carlo di Milano, nel 1967 portò in TV pazienti, parenti, medici, infermieri. Poco dopo l'INAM, il maggior ente mutualistico, stipulò una convenzione che prevedeva un rimborso di 40.000 lire per ogni seduta dialitica (comunicazione personale, 2015).

Nel 1972 veniva costituita l'ANED-Associazione Nazionale Emodializzati, che tanta parte ha avuto in questi anni nella promozione e nella difesa dei diritti dei pazienti nefropatici.

Ma la diffusione della dialisi quindi, per quanto rapida, fu per circa un quindicennio sempre inferiore alla domanda. La situazione europea di quei primi anni è illustrata nella Tabella 1. 
Tabella I. Dialisi periodica in Europa 1965-1968. ${ }^{14}$

\begin{tabular}{lrrrr}
\hline & 1965 & 1966 & 1967 & 1968 \\
\hline$N^{\circ}$ di centri dialisi & 43 & 54 & 81 & 114 \\
$N^{\circ}$ di pazienti accettati & 277 & 612 & 1163 & 2633 \\
Viventi in terapia dialitica & 160 & 295 & 621 & 1281 \\
regolare (RTD) & & & &
\end{tabular}

Tabella 2. I primi centri dialisi in Italia (fonte: ANED).

\begin{tabular}{ll}
\hline Anno & Città \\
\hline 1960 & Napoli \\
1963 & Roma \\
1964 & Milano (Croff), Catania \\
1965 & $\begin{array}{l}\text { Parma, Bologna (S. Orsola), Roma (Casa di Cura } \\
\text { Villa Gina) }\end{array}$ \\
1966 & $\begin{array}{l}\text { Roma (Policlinico Gemelli), Firenze (Careggi), } \\
\text { Padova }\end{array}$ \\
1967 & $\begin{array}{l}\text { Verona, Trieste, Udine, Genova, Lucca, Palermo, } \\
\text { Sassari }\end{array}$ \\
1968 & Genova (Pediatrico Gaslini) \\
\hline
\end{tabular}

Tabella 3. Incremento centri e pazienti in trattamento 19731980 (fonte: ANED).

\begin{tabular}{llc}
\hline Anno & Centri Dialisi & Pazienti in trattamento sostitutivo \\
\hline 1973 & 159 & 3478 \\
1074 & 178 & 4436 \\
1975 & 201 & 5558 \\
1976 & 220 & 6974 \\
1977 & 251 & 8294 \\
1978 & 277 & 9763 \\
1979 & 296 & 11285 \\
1980 & 324 & 12758 \\
\hline
\end{tabular}

Progressivamente anche gli ospedali italiani - per primi quelli universitari - cominciarono ad attrezzarsi per fornire la nuova possibilità terapeutica ai nefropatici cronici. Nella Tabelle 2 e 3 sono riportati rispettivamente gli anni di attivazione dei primi centri dialisi e l'incremento numerico centri-pazienti in trattamento dal 1973 (anno del primo Censimento ANED) al 1980. ${ }^{15}$

\section{Il ruolo della ricerca clinica e industriale}

Prima del 1960 l'interesse dell'industria alle tecniche dialitiche fu limitato a causa del mercato ancora ristretto. Nell'immediato periodo post bellico l'Europa, ed in minor misura l'America, versavano in ristrettezze finanziarie tali da non favorire investimenti in tecnologie nuove, costose, alle volte considerate bizzarre e dall'utilità incerta. Non vi furono perciò "linee di produzione" vere e proprie, anche se

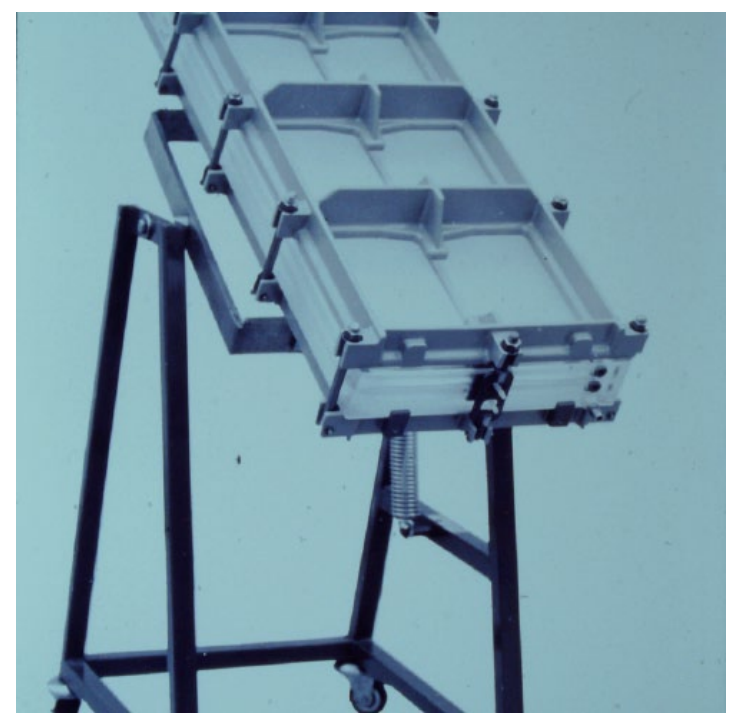

Figura 6. Dializzatore ideato da Kiil. (Arch. Bellco).

si intensificava la collaborazione progettuale tra medici, bioingegneri e rappresentanti dell'industria medicale. Gradualmente però si imposero due sistemi di dializzatore:

- sistema a flussi paralleli di Kiil ${ }^{16}$ (figure 6 e 7) che utilizzava dei fogli di cellophane sostituiti ad ogni dialisi, posti tra due piastre di materiale sintetico con la superficie interna finemente scanalata e le cui tecniche costruttive avevavo avuto una indubbia evoluzione come ad esempio nel dispositivo a piastre parallele in versione monouso (figura 8);

- sistema twin coil di Kolff a cilindro fisso con doppio avvolgimento a spirale. In materiale monouso, fu commercializzato da Baxter Laboratories a partire dal $1956 .{ }^{17}$ Kolff aveva presto abbandonato il concetto del tamburo rotante perché ritenuto troppo complesso e costoso ed insieme a Watschingher realizzato questo nuovo dializzatore, in seguito perfezionato e standardizzato (figure 9-11).

Le due tipologie ebbero per un quindicennio e più grandissima diffusione. Un vero pezzo di storia: fino a metà anni settanta con questi modelli veniva trattato oltre 1' $80 \%$ dei pazienti in dialisi periodica, ${ }^{18}$ a tal punto che nell'uso corrente per "Kiil" e "Kolff" non si intendevano i nomi dei due ricercatori ma, per antonomasia, quelli dei relativi dializzatori.

I filtri a fibre cave più costosi, ancorchè già realizzati $\mathrm{e}$ ben conosciuti, furono introdotti e impiegati solo successivamente. ${ }^{19}$

Con l'aumento delle conoscenze e dell'esperienza sugli effetti a lungo termine del trattamento sostitutivo, si tiravano i bilanci del primo decennio. Abbandonato il termine obsoleto di rene artificiale, presero a diffondersi schemi terapeutici nuovi come la dialisi trisettimanale, la dialisi 


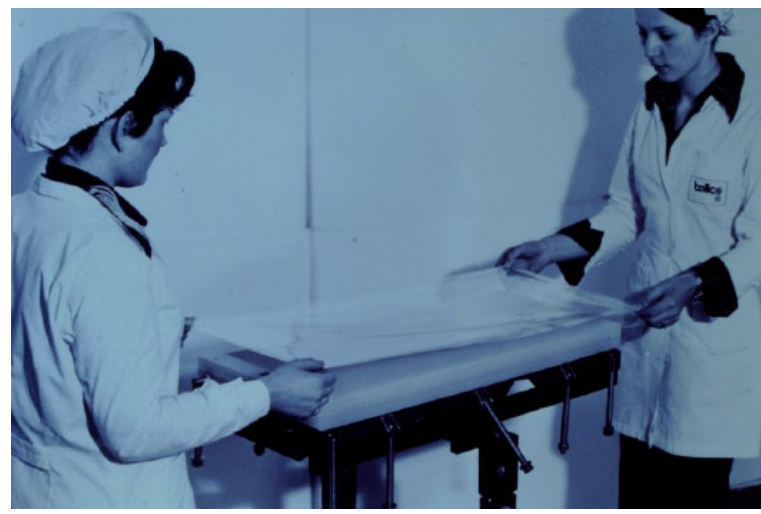

Figura 7. Allestimento di un dializzatore di Kiil.

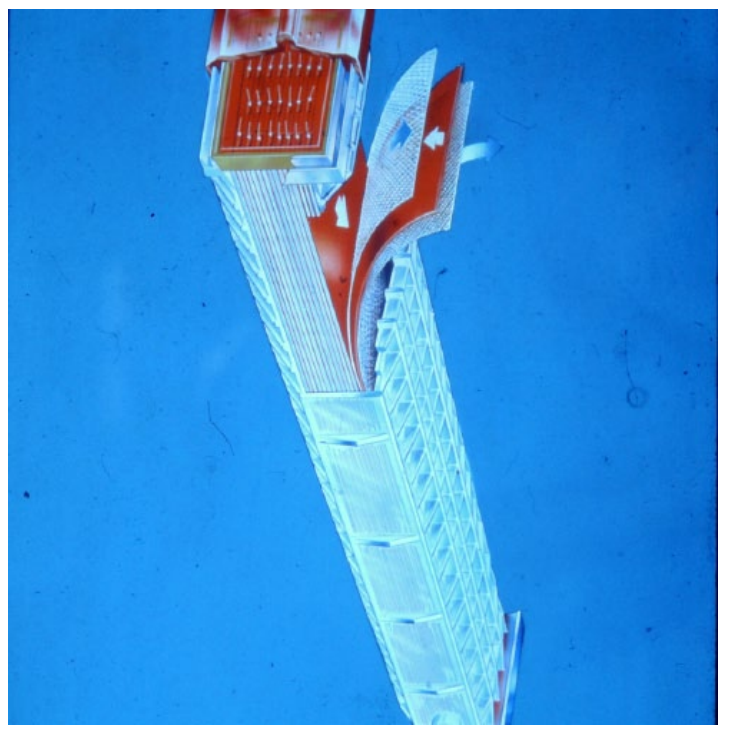

Figura 8. Evoluzione del modello Kiil in formato monouso. Raffigurazione schematica (Arch. Bellco).

breve, la bicarbonato dialisi o metodi alternativi come l'emodiafiltrazione e l'emoperfusione.

In questa decade altri risultati vennero conseguiti. Furono messi a punto un nuovo tipo di catetere peritoneale, il Tenckhoff e apparecchiature automatiche che diminuivano il rischio di contaminazione batterica. ${ }^{20}$ Anche i trapianti diventarono più facilmente realizzabili grazie ad una migliore terapia immunosoppressiva che, con l'azatioprina ed i corticosteroidi, permise un maggior controllo della risposta immunitaria del ricevente.

Anche l'industria nazionale apriva un nuovo capitolo. La rapida diffusione della dialisi nel nostro paese nella seconda metà degli anni sessanta e nella decade successiva fu resa possibile grazie ad un imprenditore attivo e perspicace, Mario Veronesi, da poco deceduto (il 12 giugno 2017), che prontamente intuì le nuove esigenze di mercato. Titolare di una azienda produttrice di deflussori per flebo e trasfusionali, tra la primavera del 1965 e del 1966,

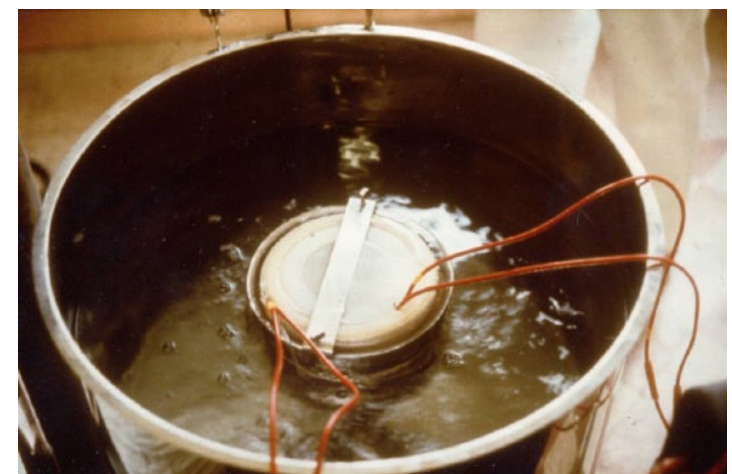

Figura 9. Dializzatore twin coil utilizzato in una apparecchiatura RSP - Ricirculating Single Pass. Fine anni sessanta (Tiziano Cerrai, per gentile concessione).

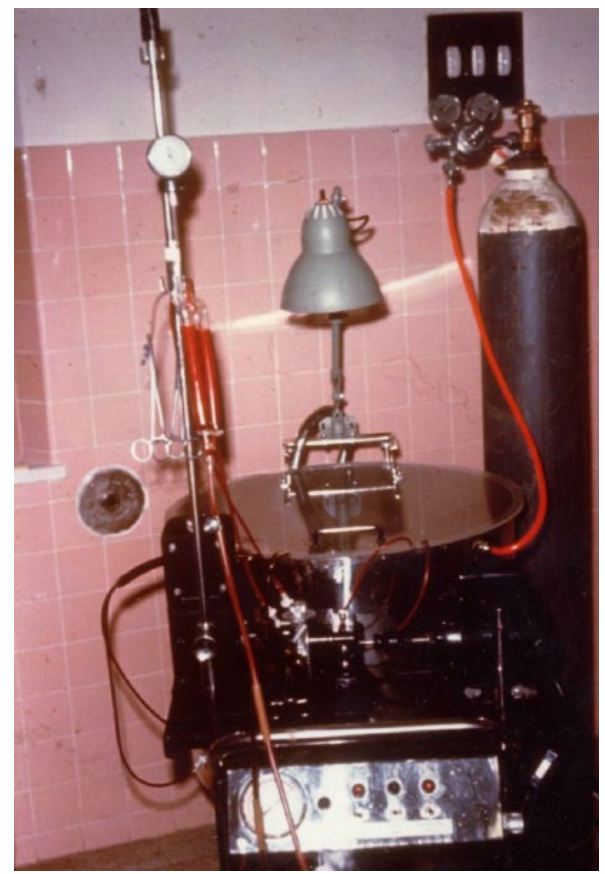

Figura 10. Apparecchiatura dializzante RSP Baxter-Travenol. Fine anni sessanta (Tiziano Cerrai, per gentile concessione).

avvalendosi della consulenza e delle indicazioni di Piero Confortini, della Patologia Chirurgica dell'Università di Padova, dove alcuni prototipi vennero collaudati e sperimentati, fu in grado di far realizzare e commercializzare un'attrezzatura completa per l'emodialisi: dissalatori, monitors, dializzatori e linee, con un marchio famoso: DASCO. Nel giugno 1966 i dati relativi all'impiego di queste prime apparecchiature furono presentati a Padova in un convegno che segnò il momento del vero boom: dopo 2 anni la DASCO, diventata leader in Europa, aveva installato oltre 1000 posti dialisi solo in Italia. Ma Veronesi non si fermò qui. Ceduta la DASCO, nel 1973 fondò la BELLCO, azienda che in quell'anno commercializzò alcuni presidi assolutamente innovativi: il modulo Unimat, 


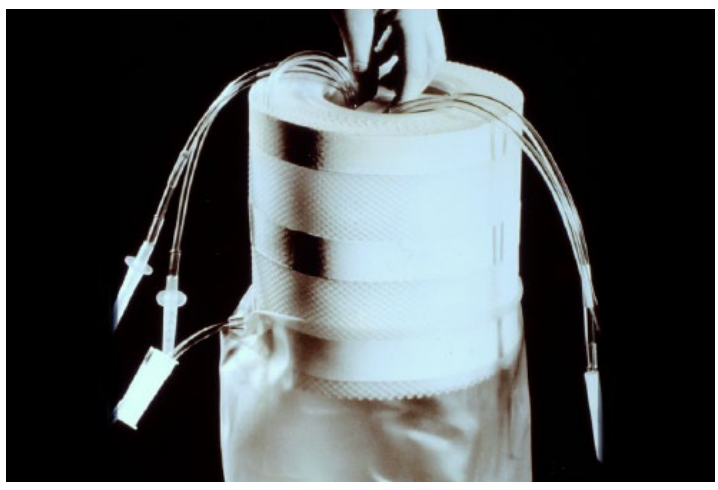

Figura I I. Modello evoluto del twin coil in uso negli anni 70 (Arch. Bellco).

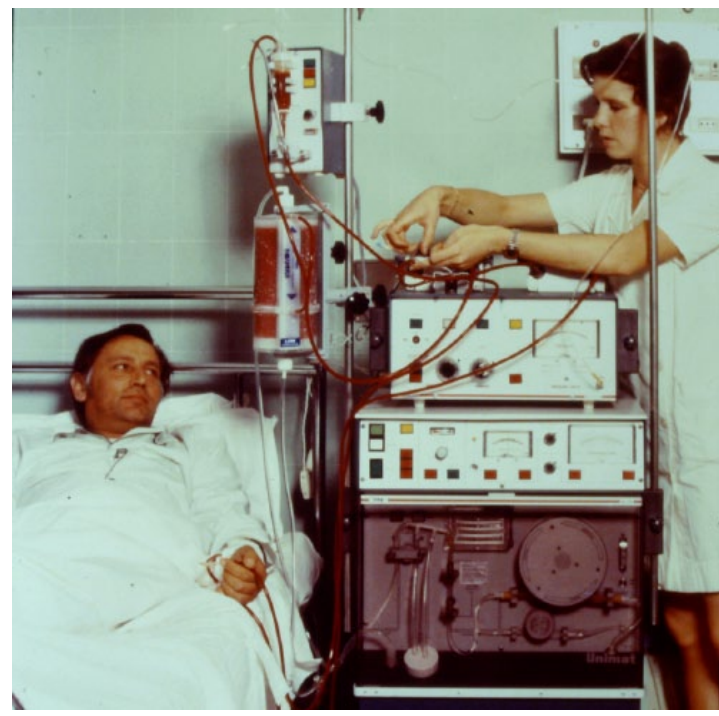

Figura I2. Primo modulo Bellco Unimat, 1973 (Arch Bellco).

la pompa peristaltica a doppia testata ed il Vita2, primo dializzatore a rotolo in single-pass (figure 12-14). ${ }^{21}$

Nel 1976 Popovich e collaboratori dimostrarono la fattibilità della dialisi peritoneale ambulatoriale continua (continuous ambulatory peritoneal dialysis, CAPD) ed invitarono il mondo industriale a sviluppare una tecnologia che rendesse la nuova tecnica largamente accessibile e sicura. La Baxter Laboratories era allora impegnata nel processo di conversione dei contenitori dei suoi prodotti dal vetro al materiale plastico. Ottenuta la licenza dalla FDA (Food and Drug Administration) per la confezione di sacche da due litri, venne commercializzato il primo sistema di scambio per la CAPD e da allora si moltiplicarono le proposte di questo sistema, nella forma manuale o automatizzata con i cosiddetti cyclers.

L'autorizzazione da parte del Federal Drug Administration all'uso del polivinile quale materiale per la costruzione delle sacche di soluzione peritoneale, diede come un notevole impulso alla CAPD. ${ }^{22}$

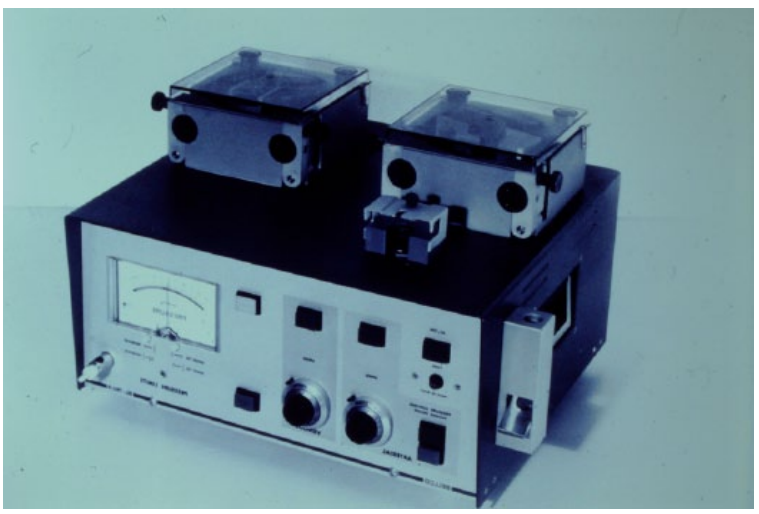

Figura 13. Pompa a doppia testata realizzata da Bellco, 1973 (Arch Bellco).

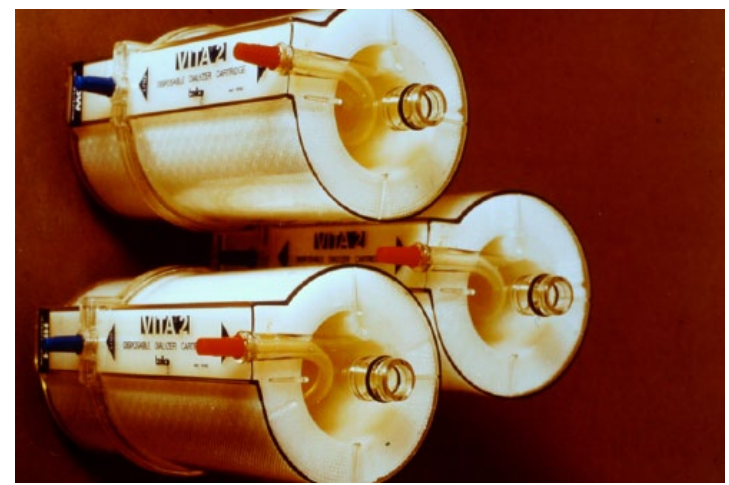

Figura 14. Dializzatore Vita 2 a single pass, Bellco 1973 (Arch. Bellco).

Nello stesso anno furono riportati i primi successi con la ciclosporina nel trattamento immunosoppressivo.

Negli anni ottanta assistiamo quindi ad un grandissimo sviluppo tecnologico del settore. Le apparecchiature diventano sempre più funzionali, sicure e versatili. La tecnologia computerizzata determina un incremento dell'efficienza, una contrazione dei tempi di trattamento ed una sempre maggiore personalizzazione. La clinica apre a successi e nuovi problemi, ${ }^{23} \mathrm{ma}$ assicurare solamente la sopravvivenza non era più ormai né sufficiente né accettabile.

Nel 1987 sono resi pubblici i primi risultati sugli effetti dell'eritropoietina. ${ }^{24}$

\section{Lo sviluppo e il consolidamento del ruolo professionale infermieristico}

I rapidi progressi della medicina e le trasformazioni socioculturali ed economiche dalla fine del secondo conflitto mondiale ad oggi, hanno portato grandi cambiamenti anche nel nursing. Il ruolo degli infermieri è cambiato nel corso degli anni in molti settori, ma specialmente nelle aree in cui la medicina e l'assistenza si sono avvalse e 
tuttora si avvalgono di tecnologie complesse e già si comincia a parlare di "competenze avanzate".

La nascita di nuovi modelli di cura introdotti dalla tecnologia, ha di riflesso determinato delle modifiche nella relazione medico-infermiera-paziente, che la letteratura anglosassone colloca in due principali fasi evolutive:

1. Da mera esecutrice di ordini e prescrizioni l'infermiera ha assunto dapprima un ruolo di assistente del medico nell'esecuzione di procedure più o meno complesse, e per far ciò ha dovuto acquisire nuove e più approfondite conoscenze compiendo un indubbio passo professionalizzante. $\mathrm{Nel}$ cogliere precocemente e positivamente questo cambiamento, Fuerst e Wolff invitavano a non distogliere dal paziente lo scopo delle attività infermieristiche: «Una delle nuove ed importanti responsabilità del nursing nei confronti del paziente consiste nell'assistere il medico nell'esecuzione di procedure diagnostiche e terapeutiche. Nelle situazioni in cui l'infermiera si trova coinvolta operando con nuove tecnologie o facendo ricerca, essa dovrà escogitare con giudizio e originalità nuove strategie di supporto al paziente, fino ad allora sconosciute $» ; 25$

2. Un secondo rilevante cambiamento si è verificato man mano che si sono ampliate le conoscenze di base, strumento grazie al quale l'infermiera ha potuto definire meglio le proprie funzioni, in rapporto alla globalità del paziente come persona ed alla sua individualità.

Nel corso degli anni sessanta era prevedibile che le infermiere sarebbero state coinvolte in un utilizzo rilevante della tecnologia, anche in considerazione del fascino (e della gratificazione) che poteva esercitare la consapevolezza di operare in un campo considerato di dominio maschile.

La sfida venne raccolta da diversi e autorevoli pensatori dell'infermieristica che offrirono chiavi di lettura precognitive per allora, attuali per oggi:

- assistere il medico o possedere anche rilevanti abilità tecniche va visto sempre come elemento complementare al ruolo tradizionale del nursing, che nel rapporto interpersonale con il paziente deve trovare la misura dell'utilizzo delle conoscenze e delle competenze; 26

- «ogni infermiera dovrà usare il giudizio in ogni situazione in cui l'uso di nuove tecnologie abbia effetti deleteri sul paziente come persona, controbilanciando questi effetti mediante buone relazioni interpersonali. È perciò necessario con cura i possibili influssi sulla personalità e limitare tutto ciò che nel lavoro è spersonalizzante»; ${ }^{27}$
- l'aumentata motivazione alla ricerca di tecnologie complesse deve stimolare un utilizzo creativo dell'elettronica con lo scopo di ottenere trattamenti curativi ancor più personalizzati. ${ }^{28}$

Si è molto sensibile al problema anche nel mondo infermieristico italiano. Nel 1968 la Consociazione Nazionale Infermiere essionali Assistenti Sanitarie e Vigilatrici d'Infanzia (oggi CNAI) titolò «La professione infermieristica in un'era tecnologica» il $\mathrm{VII}^{\circ}$ Congresso Nazionale. Vi possiamo cogliere tensioni ed interrogativi che nulla hanno da invidiare all'autorità dei teorici anglosassoni: «La formulazione del tema, - sottolineava Marina Caruana, presidente della Consociazione - non vuole approdare a soluzioni meramente critiche. È nostro intendimento primario fare il punto sulla situazione, esaminare cioè come la nostra professione debba accettare lo spirito, gli impulsi e gli strumenti dell'epoca in cui viviamo, un'epoca tutta protesa verso un continuo approfondimento tecnologico;... certo, introducendo un termine come "tecnologia" nel tema della professione infermieristica, si sente un certo stridore, quasi una contraddizione di termini. È vero, infatti che la professione infermieristica richiede una preparazione ed una formazione ben più complesse e difficili, ma è altrettanto vero che alla base di una tale professione, nella sua anima stessa, risiedono doti squisitamente umane»..$^{29}$

Questo dunque è lo scenario in cui si inserisce la nascita ed il progressivo espandersi del nursing nefrologico. Quest'area di attività infermieristica si trova ancor oggi in una posizione particolare: deve continuamente ripensare e ridefinire, in un costante clima di cambiamenti, il proprio ambito di specificità, di autonomia e di interdipendenza.

Non c'è dubbio che le infermiere che hanno prestato la loro opera in questo primo periodo hanno contribuito a produrre molte idee utilizzate poi per modificare in senso migliorativo i primi dispositivi di dialisi. Se il rene artificiale è da annoverare tra le invenzioni del ventesimo secolo, sviluppatasi soprattutto dalla collaborazione tra il mondo medico e l'industria, le infermiere del ventesimo secolo sono state le artefici della sua progressiva applicabilità al paziente, mediante l'attenta osservazione di tutto ciò che accadeva nel corso di questo processo. Il loro ruolo che consisteva nel fare da sponda agli inventori è ora oggetto da parte degli storici del nursing. ${ }^{30}$

Ben presto esse si resero consapevoli della necessità di modificare la loro funzione di semplici assistenti del medico per assumere un nuovo ruolo. Dapprima utilizzavano le conoscenze scientifiche dedicandosi esclusivamente alle tecniche dialitiche (allestimento e messa in funzione del rene, preparazione del bagno di dialisi) ed al controllo dei parametri fisici del paziente, ma ben presto cominciarono a dedicarsi più intensamente ad approfondire l'aspetto relazionale. 
Le testimonianze che si riportano non sono che alcune fra le numerosissime che potrebbero essere raccontate e che rappresentano, singolarmente considerate, parti di una grande storia che ha preso forma, sviluppo, realizzazione attraverso un ideale filo rosso che le teneva unite in un indissolubile legame di consapevolezza, responsabilità, volontà di progresso professionale, scientifico culturale, umano.

\section{Nursing nefrologico: testimonianze di storia vissuta}

- «Negli anni seguenti, diffusasi la notizia che il rene artificiale poteva salvare la vita, le ultime speranze di sopravvivenza portarono alcuni pazienti ed i loro familiari ad affannose ricerche di un posto dialisi anche molto lontano dalla propria residenza. A Parma nel 1966 venivano trattati pazienti di Torino, Brescia, Trento, Pescara e Roma. Il primo centro dialisi era stato ricavato nei locali dello scantinato, dove fu messo in funzione un impianto centralizzato per la distribuzione del liquido di dialisi a ciclo continuo, formato da due cisterne di circa 400 litri ciascuna. Una serviva per la preparazione del liquido di dialisi e l'altra per la distribuzione ai posti letto. Le sedute duravano 12-14 ore». Aurelia Castagnoli (Comunicazione personale manoscritta, dicembre 1995).

口 «Grazie alla capacità ed alla dedizione di molti infermieri, unita a quella dei medici provenienti da diversi settori della medicina, nonché alla stretta collaborazione instaurata con i tecnici di alcune ditte italiane, le apparecchiature si sono evolute, sono state sperimentate, e continuamente perfezionate... Non esistevano ad esempio dispositivi per il controllo delle perdite ematiche o per la rilevazione di aria nel gocciolatore venoso, per cui tanti controlli erano affidati alla sorveglianza visiva continua. $\mathrm{Al}$ termine della dialisi era compito degli infermieri lo smontaggio delle apparecchiature di Kiil, il lavaggio delle piastre, il nuovo allestimento con le membrane di cellophane, la prova di tenuta, l'immissione di soluzione sterilizzante che veniva lasciata in situ fino al mattino successivo. La durata della dialisi era di 12 ore e tali attività si svolgevano sempre nelle ore notturne. Frequenti erano gli incidenti tecnici. In particolare i centri non disponevano di gruppi elettrogeni e le pur rare interruzioni di corrente creavano problemi cui il personale infermieristico doveva far fronte con tempestività e sicurezza. In primo luogo bisognava garantire l'eparinizzazione ed il ricircolo manuale del sangue e, se l'interruzione diventava troppo prolungata, reinfondere il paziente. Frequentissimi erano i casi di rottura della membrana dializzante che potevano comportare, specie con ultrafiltrazione elevata, perdite ematiche cospicue. La sostituzione del dializzatore richiedeva tempi molto lunghi: 30 minuti se si disponeva di un dializzatore di riserva, uno a due ore se si doveva provvedere ad una nuova sterilizzazione». Enzo Giannoni (Coordinatore Dialisi Monna Tessa, Firenze $)^{31}$

口 «Non si poteva mai abbassare la guardia... noi abbiamo imparato dalla nostra pratica giornaliera e le nostre orecchie erano abituate a reagire a suoni diversi: l'eccessiva compressione sulle linee della pompa arteriosa indicava un flusso ematico insufficiente, un colpo di tosse stizzoso era un chiaro segnale di embolia gassosa». Franz Techert(Coordinatore Servizio Dialisi di Giessen, Germania) ${ }^{32}$

\section{L'assistenza infermieristica in emodialisi 1962-1966 a Seattle di Carolyn Hopf (Infermiera, Thun, Svizzera) $)^{33}$}

Il mio primo incontro con la dialisi avvenne nell'Ospedale Universitario dello stato di Seattle, nello stato di Washington (USA) nel 1962 con uno dei primi pazienti del dottor Scribner. Seattle aveva allora 2 centri dialisi, nell'Ospedale Universitario e nell'Ospedale Svedese, i primi per il trattamento dell'IRC negli USA.

Le attrezzature erano molto limitate pertanto fu istituito un comitato di selezione dei pazienti che si era dato delle linee guida molto rigide per stabilire l'eleggibilità dei pazienti alla dialisi. Il paziente veniva ammesso alle seguenti condizioni:

- avere un'età inferiore ai 40 anni

- avere un impiego lavorativo

- essere un membro utile per la sua comunità

- avere buone possibilità cliniche di tollerare la dialisi

- accettare positivamente la terapia

Ciò significava ad esempio che una persona di sesso maschile che avesse un'occupazione e famiglia aveva maggiori possibilità di essere accettata nel programma di una giovane casalinga con figli.

La mia personale esperienza a Seattle fu limitata ad una sola notte, quando venni chiamata a sostituire nella piccola Unità 
Dialisi l'infermiera di turno che si era ammalata. Ho dovuto così confrontarmi con gli ingombranti macchinari dietro cui stavano nascosti tutti i pazienti! Quell' unica notte tuttavia destò a tal punto la mia curiosità che, alcuni anni dopo, quando mi recai per un'esperienza di lavoro in Canada, chiesi di essere assegnata alla piccola Unità di Ricerche Nefrologiche del Vancouver General Hospital, dove veniva ancora impiegata una vecchia apparecchiatura dialitica a prima vista simile a un enorme bollitore che per l'uso doveva prima essere riempita con alcuni litri di sangue. Era adatta a trattare transitoriamente pazienti affetti da insufficienza renale acuta (IRA) ma non i malati cronici. La commercializzazione delle vasche per il dialisato e del rene di Kiil modificato da Meltec usato a Seattle e migliori conoscenze sulla chirurgia dell'inserzione dello shunt di Scribner resero in seguito possibile il trattamento dei pazienti cronici. Il Meltec-Kiil era un dializzatore riusabile formato da tre piastre di polietilene scanalato. Su ciascun lato della piastra mediana l'infermiera o il tecnico stendeva due fogli di cellophane attraverso i quali scorreva il sangue, mentre il dialisato fluiva in senso opposto tra il lato del polipropilene ed il cellophane adiacente. Il sistema era tenuto insieme da una montatura di alluminio fissata con viti a bullone. Il dializzatore veniva riempito di soluzione salina e dopo la dialisi le membrane di cellophane e le linee sangue venivano gettati e le piastre trattate con formalina. La vasca conteneva il bagno di dialisi riscaldato e formato da acqua e normali quantità di sali misurate e mescolate dall'infermiera o dal tecnico in quantità in misura sufficiente per 10-14 ore. Il contenuto del bagno di dialisi era registrato su una scheda dal responsabile della preparazione.

Il chirurgo posizionava lo shunt di Scribner nell'avambraccio o nella gamba del paziente usando tubi di teflon e silastic. Le estremità erano fissate insieme all'esterno mediante un connettore maschio-femmina fissato da anelli metallici e con un ponte di cerotto. La procedura per la messa in dialisi del paziente era completamente asettica. L'infermiera indossava maschera e guanti sterili ed il suo assistente la maschera. La fasciatura veniva rimossa dallo shunt del braccio o dalla gamba, al di sotto dei quali veniva posto un telo sterile. Si eseguiva una disinfezione, si rimuoveva il cerotto dalle connessioni, lo shunt veniva clampato su ciascun lato ed i connettori separati con uno speciale forcipe. Il lato arterioso dello shunt veniva connesso alla linea arteriosa, poi si faceva defluire in un contenitore sterile la soluzione di riempimento. Quando la linea venosa cominciava a diventare rosata, veniva collegata allo shunt ed aveva inizio la dialisi. L'unica garanzia di sicurezza durante le 8-14 ore della dialisi era costituito dalla presenza dell'infermiera! Ogni 30 minuti venivano effettuati e registrati sulla scheda dialitica i seguenti controlli:

- pressione arteriosa e frequenza;

- tempo di coagulazione del paziente e del circuito extracorporeo per determinare il fabbisogno di eparina ed eventualmente di protamina da somministrare arteriosa;

- velocità di flusso ematico mediante introduzione di $1 \mathrm{cc}$ di aria nella linea venosa tra il dializzatore ed il gocciolatore venoso con aggiustamento della pompa peristaltica, se necessario;

- velocità di flusso del dialisato. Il liquido di dialisi veniva misurato allo scarico facendolo defluire per un minuto in un contenitore graduato;

- ultrafiltrazione: era possibile solo applicando un morsetto a vite sulla linea venosa. Il solo controllo realizzabile del fluido rimosso era il peso reale del paziente cosicché questi doveva salire su una bilancia a intervalli regolari. Ciò spesso causava ipotensione e l'infermiera doveva sempre avere pronta della soluzione fisiologica da infondere;

- perdite ematiche: potevano essere solamente rilevate "a vista" attraverso uno spezzone di vetro trasparente inserito nel tubo di scarico del dialisato;

- presenza di schiuma o microbolle nel sangue, abbastanza frequente, dovuta a connessioni delle linee ematiche ancora imperfette.

Altre competenze infermieristiche erano la sostituzione del gocciolatore venoso in corso di dialisi, la somministrazione di dosi supplementari di eparina per prevenire la coagulazione, la sostituzione dello spezzone della linea arteriosa inserito nella pompa in caso di rottura (la qualità del materiale era assai diversa da quella attuale).

L'ematocrito dei pazienti era spesso assai basso ed essendo sconosciuta l'eritropoietina si praticavano frequenti trasfusioni di emazie concentrate. I prelievi per gli esami ematici venivano effettuati pre e post dialisi. I risultati fuori dai limiti considerati normali per un paziente dializzato dovevano essere corretti mediante un prolungamento delle sedute dialitiche e maggiori attenzioni alla dieta. Poiché i dializzatori erano molto meno efficienti degli attuali, il paziente era obbligato a seguire una dieta particolarmente rigida.

Mezzora prima della fine della dialisi il Kiil veniva inclinato con il gocciolatore posto verso il basso per favorire il flusso del sangue verso la linea venosa. Allo stacco veniva clampato il tratto arterioso dello shunt. Scollegata la linea arteriosa, vi si insufflava lentamente dell'aria fino allo svuotamento di tutto il filtro. Chiuso anche il tratto venoso dello shunt e scollegata la linea venosa, le due estremità del by pass venivano unite con l'avvertenza di lasciare nel tratto arterioso una minuscola bolla d'aria. L'infermiera rilasciava contemporaneamente le due clamp e poteva così osservare la velocità di flusso nello shunt quando la bolla lo percorreva ed entrava nella vena del paziente. Si applicavano poi delle garze sterili ed un bendaggio lasciando fuoriuscire una piccola parte della cannula per permettere al paziente di individuare precocemente una eventuale coagulazione.

Se lo shunt era coagulato il paziente doveva venire in ospedale dove il medico o un'infermiera opportunamente preparata provvedeva alla disostruzione. Si tentava di estrarre il coagulo con una siringa e se ciò non era possibile si iniettavano rapidamente $30 \mathrm{cc}$ di soluzione fisiologica tentando una rimozione forzata del coagulo. In caso di insuccesso era necessaria una disostruzione meccanica.

Le pazienti di sesso femminile venivano dializzate durante il giorno ma la maggior parte dei pazienti erano uomini o bambini in età scolare che iniziavano la loro giornata dopo aver fatto 10-14 ore di dialisi durante il pomeriggio e la notte. Al mattino facevano colazione e poi andavano a scuola o al lavoro. Abbiamo fatto molta strada da allora! 


\section{Dalla sopravvivenza alla riabilitazione}

Come si è visto, gli anni sessanta si aprirono all'insegna di grandi speranze, dopo i primi risultati di sopravvivenza a lungo termine nei pazienti affetti da IRC.

Gli elementi significativi che caratterizzano il nursing nefrologico di quel periodo, in progressiva espansione discendono da questi obiettivi: sopravvivenza, prevenzione delle complicanze, educazione e riabilitazione del paziente, conoscenza e perfezionamento delle tecniche.

Il momento segnava l'inizio di una preponderante presenza infermieristica nell'erogazione della terapia sostitutiva e nella conduzione delle lunghe sedute dialitiche che, allora bisettimanali, duravano 10-14 ore. Ma la nuova realtà con i suoi positivi e brillanti risultati portava anche nuove e più complesse problematiche e «come sorveglianti, educatrici e consigliere dei pazienti in dialisi, le infermiere erano particolarmente consce degli stress fisici, emozionali e sociali che molti pazienti sperimentavano malgrado la dialisi salvasse loro la vita e procurasse loro dei benefici». ${ }^{34}$

JoAnn Albers, la prima caposala dell'Unità Dialisi di Seattle ricorda che si era stabilito un formidabile esprit de corps con i pazienti al punto tale che «i medici dipendevano da noi per la maggior parte delle interazioni nella sfera psicosociale con essi. A tal punto ci prendevamo cura di loro che conoscevamo tutto anche delle loro famiglie». ${ }^{35}$

Molti problemi tecnici persistevano ed ai contributi della loro risoluzione contribuivano intuizione ed ingegno in un campo nel quale ancora nessuno poteva definirsi esperto.

In Italia a partire dal 1968 fu data agli infermieri professionali, purtroppo solo per poco, la possibilità di specializzarsi con la frequenza a corsi in nefrologia e tecniche dialitiche, organizzati presso scuole infermieri professionali o istituti universitari, prima fra tutte la Clinica Nefrologica dell'Università di Parma diretta dal prof. Luigi Migone. Questi corsi (nella terza parte se ne parlerà più ampiamente) erano ben distanti dagli attuali modelli orientati al nursing e sarebbero ora solo parzialmente proponibili, ma all'epoca hanno consentito a molti infermieri di acquisire conoscenze e abilità tecniche tali da permettere un loro rapido inserimento nel mondo del lavoro, a seguito del continuo incremento numerico dei pazienti da trattare.

La continuità della dialisi fu messa in crisi nel 1974 a causa di vistosi ritardi degli enti pubblici nel pagamento del materiale ai fornitori. Esaurite le scorte, si dovette ricorrere anche al riutilizzo dei dializzatori, operazione per la quale nessuno era attrezzato.

Durante la decade degli anni settanta si sviluppò in maniera notevole la pratica dell'emodialisi domiciliare, anche come mezzo di migliore riabilitazione. Diretto responsabile dell'insegnamento al paziente era il personale infermieristico che aveva non solo il compito di insegnare la manualità tecnica ed il funzionamento delle apparecchiature, ma anche e soprattutto quello di stabilire un valido rapporto psicologico, indispensabile per l'acquisizione da parte del paziente e del suo partner delle condizioni di fiducia nelle proprie capacità di apprendere e di far fronte alle emergenze.
Gli scenari che si aprivano nel corso degli anni settanta comportavano sfide di tutti i tipi: cliniche, scientifiche, sociali, economiche. Non era ancora stato istituito il Servizio Sanitario Nazionale e ogni sorta di problemi quotidianamente doveva essere affrontata in diversi contesti.

L'Emilia Romagna per mezzo dell'AROER Associazione Regionale Ospedali Emilia Romagna ebbe il merito di organizzare nel 1975 un grande convegno nazionale "Prevenzione e riabilitazione nell'insufficienza renale: esperienze, problemi e prospettivi," con oltre 500 partecipanti: medici, amministratori, politici, personale dei servizi dialisi, associazioni, pazienti nefropatici. L'evento merita di essere ricordato perché venne approntato con grande accuratezza: fu predisposto e distribuito in 4000 copie un documento preparatorio contenente dati epidemiologico-statistici rilevanti per l'epoca (tabella 4) e informazioni utili per i partecipanti che potevano quindi prepararsi in maniera adeguata.

Per quanto ci è dato sapere fu anche la sede in cui gli infermieri italiani attraverso la voce di Aurelia Castagnoli, portarono il loro primo contributo professionale citabile in letteratura. ${ }^{36}$

Gli anni settanta si concludevano segnando la fine di un'emergenza durata quasi vent'anni: poter effettuare il trattamento a casa o non lontano da essa con apparecchiature efficienti e sicure, affidate a personale infermieristico tecnicamente capace, anche se preparato grazie all'insegnamento di altri colleghi più che con la frequenza a corsi specialistici in progressiva disattivazione, non costituiva più per il paziente nefropatico un'incognita.

\section{L'epatite nei centri dialisi}

Le tecnologie dialitiche comportavano anche dei rischi professionali sui quali le conoscenze non erano ancora tali da dettare le idonee misure preventive o precauzionali e non esisteva ancora la vaccinazione. Durante negli anni sessante e settanta altissima fu l'incidenza di infezione da HBV tra i pazienti e il personale medico e infermieristico: in Europa ben 6700 operatori, dal 1969 al 1975 contrassero l'epatite, malattia mortale per un centinaio di essi. ${ }^{37}$ Un'indagine italiana condotta su 178 centri e 3780 operatori nel 1986 ha rilevato ben 610 casi di infezione di cui 453 sfociati in malattia. ${ }^{38}$

Alcuni centri furono costretti a chiudere, mentre veniva sostenuta la necessità di controlli addirittura settimanali per la ricerca anticorpale. ${ }^{39}$

\section{L'evoluzione del nursing: self-care e qualità}

Nel corso degli anni ottanta il ruolo degli infermieri in ambito nefrologico si è ulteriormente espanso. Le opportunità di scelta e la flessibilità di impiego e applicazione delle terapie sostitutive, incluso il trapianto, hanno imposto nuovi modelli di cura e assistenza orientati al raggiungimento di 
Tabella 4. Centri dialisi, posti e pazienti in trattamento nelle varie regioni e in Italia (dati espressi in cifre assolute e per milione di abitanti). Situazione al 3I dicembre 1974 (AROER, 1975).

\begin{tabular}{|c|c|c|c|c|c|c|c|c|}
\hline \multirow[t]{2}{*}{ REGIONI } & \multirow[t]{2}{*}{ Abitanti } & \multirow[t]{2}{*}{ Dens. } & \multicolumn{2}{|c|}{ Centri Dialisi } & \multicolumn{2}{|c|}{ Posti Dialisi } & \multicolumn{2}{|c|}{ Pazienti in Emodialsi } \\
\hline & & & Totale & $\begin{array}{l}\text { Per mil. } \\
\text { di ab. }\end{array}$ & Totale & $\begin{array}{l}\text { Per mil. } \\
\text { di ab. }\end{array}$ & Totale & $\begin{array}{l}\text { Per mil. } \\
\text { di ab. }\end{array}$ \\
\hline Piemonte & 4,462 & 176 & 17 & 3,81 & 184 & 41,24 & 371 & 83,15 \\
\hline Valle d'Aosta & 0,110 & 34 & - & - & - & - & - & - \\
\hline Lombardia & 8,630 & 362 & 26 & 3,01 & 319 & 36,96 & 912 & 105,68 \\
\hline Trentino A.A. & 0,848 & 62 & 2 & 2,36 & 22 & 25,94 & 62 & 73,11 \\
\hline Veneto & 4,168 & 227 & 14 & 3,36 & 175 & 41,99 & 448 & 107,49 \\
\hline Friuli V.G. & 1,223 & 156 & 5 & 4,09 & 52 & 42,52 & 108 & 88,31 \\
\hline Liguria & 1,862 & 344 & 8 & 4,29 & 76 & 40,79 & 169 & 90,71 \\
\hline Emillia-Rom. & 3,883 & 175 & 9 & 2,32 & 119 & 30,73 & 355 & 91,66 \\
\hline Toscana & 3,502 & 162 & 18 & 5,14 & 197 & 56,25 & $4 \mid 4$ & || $8,2 \mid$ \\
\hline Marche & 1,366 & $|4|$ & 5 & 3,66 & 42 & 30,75 & 103 & 75,40 \\
\hline Umbria & 0,780 & 92 & 3 & 3,85 & 22 & 28,21 & 80 & 102,56 \\
\hline Lazio & 4,740 & 276 & 20 & 4,22 & 186 & 39,24 & 331 & 69,83 \\
\hline Abruzzi & 1,179 & 109 & 5 & 4,24 & 46 & 39,02 & 82 & 69,55 \\
\hline Molise & 0,322 & 73 & - & - & - & - & - & - \\
\hline Campania & 5,118 & 376 & 17 & 3,32 & 146 & 28,53 & 271 & 52,95 \\
\hline Puglia & 3,628 & 188 & 10 & 2,76 & 147 & 40,52 & 276 & 76,07 \\
\hline Basilicata & 0,607 & 61 & I & 1,65 & 12 & 19,77 & 26 & 42,83 \\
\hline Calabria & 1,996 & 132 & 4 & 2,00 & 50 & 25,05 & 134 & 67,13 \\
\hline Sicilia & 4,724 & 184 & II & 2,33 & 109 & 23,07 & 243 & 51,44 \\
\hline Sardegna & 1,495 & 62 & 3 & 2,01 & 21 & 14,05 & 51 & 34,11 \\
\hline ITALIA & 54,643 & $|8|$ & 178 & 3,25 & 1.925 & 35,22 & 4.436 & 81,18 \\
\hline
\end{tabular}

alti livelli di qualità con un utilizzo razionale delle risorse, in termini che sogliono essere definiti come elevati benefici a bassi costi. Tutto ciò ha comportato:

- $\quad$ per il paziente un potenziamento del self-care e di una maggior responsabilizzazione e coinvolgimento nelle decisioni (sui modi ed tempi del trattamento);

- per l'infermiere l'utilizzo in modo massiccio di una potente leva di adattamento professionale: la ricerca, in ambiti consueti come tecniche dialitiche, ma soprattutto nuovi, in relazione all'attenzione posta alla qualità dei servizi sanitari. Di primario interesse sono così diventati: l'introduzione e la valutazione di nuove opzioni di trattamento sostitutivo, la revisione ed il controllo dei protocolli assistenziali, l'individuazione di indicatori della qualità della vita. ${ }^{40}$

Il continuo aumento della popolazione dialitica ha comportato un più gravoso impegno infermieristico per la necessità di far fronte a problematiche del paziente sempre più complesse spesso non limitate all'ambito nefrologico. Questa situazione ha determinato spesso una diminuzione della gratificazione professionale ed un maggiore incidenza del burnout negli infermieri. ${ }^{41}$

Un secolo di nursing nefrologico proseguirà nel prossimo numero con la terza parte: "Dagli anni 90 a oggi. II nursing nefrologico nell'epoca della globalizzazione”.

\section{Riferimenti bibliografici}

1. McCormick TR. Ethical Issues in Caring for Patients with Renal Failure. ANNA J 1993; 20: 549-555.

2. Scribner BH, Buri R, Caner JE, et al. The treatment of chronic uremia by means of intermittent hemodialysis. A Progress Report. J Am Soc Nephrol 1998; 9: 719-726.

3. Brescia MJ, Cimino JE, Appell K, et al. Chronic hemodialysis using venipuncture and a surgically created arteriovenous fistula. N Engl J Med 1966; 275: 1089-1092.

4. Scribner BH, Quinton WE. Blagg CR, et al. Choice of patients to undergo maintenance hemodialysis. In: Treatment of chronic uremia (ed. Eurospital, Trieste 1971). Practical manual for doctors. Seattle Artifical Kidney Center, Seattle, 1967: 9-16.

5. Scribner BH e Babb AL. Chronic Hemodialysis in Seattle 1960-1966. Dialysis \& Transplantation 1986; 15:33-45

6. Scribner BH. Foreword to the first edition. In: Drukker W, Parsons FM e Maher FM (eds). Replacement of renal function by dialysis. Boston: Martinus Nijhoff Publishers, 1983.

7. Alexander S. They decide who lives, who dies. Life Magazine, 9 Novembre 1962; 53: 102-124.

8. Campanacci L, Romagnoli GF e Huber W. Criteri di selezione dei pazienti da avviare all'emodialisi periodica. Atti della Tavola Rotonda: Aspetti medici, sociali, giuridici ed organizzativi dell'emodialisi periodica, Venezia 16 aprile 1972: 11-21.

9. Introna F. L'emodialisi periodica: problemi medico-sociali e medico-legali. Atti della Tavola Rotonda: Aspetti medici, sociali, giuridici ed organizzativi dell'emodialisi periodica, Venezia 16.4.1972: 117-127. 
10. Shaldon S. The Self-Service Approach. Dial Transplant 1986; 15: 19.

11. Merril J, Schupak E, Cameron E, Hampers CL. Hemodialysis in the home. Journal of the American Medical Association 1964; 190: 468-470. https://www.ncbi.nlm.nih. gov/pubmed/?term=MERRILL\%20JP\%5BAuthor\%5D\&ca uthor=true\&cauthor_uid $=14197998$

12. Alwall N. Historical Perspective on the Development of Artificial Organs. (Lecture). Fifth World Congress of the ASAIO, Chicago, 6 October 6, 1985. Lund: Gambro, 1986, pp.16-17.

13. Testimone di una operazione editoriale di basso profilo, completamente anacronistica ed inconsapevole di quanto stava accadendo e di una realtà in pieno sviluppo, è la citazione che segue, del 1973. Allora mentre i dializzati erano ormai già 5000 distribuiti in 1500 posti sul territorio nazionale, un medico generico difficilmente avrebbe potuto ben consigliare un paziente nel quale avesse riscontrato uno stato di avanzata insufficienza renale, se si fosse basato su un diffuso manuale che, giunto alla sesta edizione completamente riveduta e ampliata, al capitolo "Nefriti croniche" così dissertava: «Alle tecniche terapeutiche eccezionali, applicabili solo in speciali ambienti, appartengono il rene artificiale, la dialisi peritoneale, l'exanguinotrasfusione, il trapianto renale ecc. Il rene artificiale, però, può essere anche praticato ambulatoriamente; la dialisi viene fatta ad intervalli più o meno brevi di tempo, ma la sua efficacia diminuisce con il maggior numero di applicazioni. Procedimenti assai più semplici sono la perfusione intestinale, la perfusione stomacale, la perfusione del colon, la perfusione prolungata della vescica.» Tratto da: Messini M e Meccoli V. Compendio di terapia delle malattie interne. Roma: SEI VI, 1973, p.429.

14. Drukker W, Schouten WAG e Alberts C. Regular on report dislysis treatment in Europe IV, 1968. Proc Eur Dial Transpl Assoc 1969; 5:3.

15. ANED - Associazione Nazionale Emodializzati. Censimento dei Servizi Dialisi in Italia, 1972 e seguenti. http://www.anedonlus.it/guide-manuali/censimento-dei-servizi-di-dialisi-initalia-aggiornato-al-31122016/ (consultato: 21 marzo 2018)

16. Kiil F. Development of a parallel flow artificial kidney in plastics. Acta Chir Scandinavica. 1960; 253: 143-146.

17. Kolff W e Watschinger B. Further development of a coil kidney. J Lab Clin Med 1956; 47: 969-977.

18. Gurland HJ, Kessel M, Massina MA, et al. Emodialisi \& Dialisi Peritoneale. Padova: Piccin, 1973, p.98.

19. Steward RD, Lipps BJ, Barretta ED, et al. Hedialysis with the capillary kidney. Trans Am Soc Artif Intern Organs. 1968; 14: 121-125.

20. Tenckhoff H e Schechter H. A bacteriologicallly safe peritoneal dialysis acces device. Trans ASAIO 1968; 14: 181-183.

21. Veronesi M. L'inizio della dialisi in Italia. Atti Seminario: Il paziente in dialisi dopo 25 anni. Milano: Wichtig, 1996.

22. Popovich RP, Moncrief JW, Nolph KD, Ghods AJ, Twardowsky ZJ e Pyle WK. Continuous ambulatory peritoneal dialysis. Ann Intern Med 1976; 88:449-456.

23. Cameron JS. The 1970s and 1980s: new technical advances and some new problems. In: Cameron JS. History of the treatment of renal failure by dialysis. Oxford: Oxford University Press, 2002, pp.229-247.
24. Eschbach JW, Egrie JC, Downing MR, et al. Correction of anemia of end-stage renal disease with recombinant human herytropoietin: Results of a combined phase I and II clinical trial. N Eng J Med 1987; 316:73-78.

25. Fuerst EV e Wolff LV. Fondamentals of nursing: The humanities and the sciences in Nursing. Philadelphia: JB Lippincott, 1959.

26. Montag ML e Swenson RPS. Fondamentals in nursing care. Philadelphia: WB Saunders, 1959.

27. Peplau HE. Automation: will it change nurses, nursing, or both? In: Technical innovations in health care: Nursing implications. New York: American Nurse's Association, 1962.

28. Downs FS. Technical innovation and the future of the nursing-patient relationship. In: ANA Clinical Sessions. New York: ACC, 1960.

29. Caruana M. Relazione d'apertura. In: Atti del VII Congresso Nazionale della Consociazione Nazionale Infermiere Professionali, Assistenti Sanitarie e Vigilatri-ci d'Infanzia: La professione infermieristica in un'era tecnologica. Roma, 26-29 ottobre 1968.

30. Sandolowsky M, Hiestand W e Fairman G. The machine in the sick room. Am J Nephrol 1997; 17: 299-303.

31. Giannoni E. L'approccio infermieristico alla dialisi in Italia prima degli anni '70. In: Atti del XII Congresso Nazionale Edtna-Erca 1943-1993: la dialisi per cinquant'anni al servizio dell'uomo, Brescia 18-20 ottobre 1993.

32. Techert F. L'evoluzione nel tempo del nursing nefrologico: nuove funzioni, nuove rsponsabilità per l'infermiere. In: Atti XII Congresso Nazionale Edtna-Erca: 1943-1993: la dialisi per cinquant'anni al servizio dell'uomo, Brescia 18-20 ottobre 1993.

33. Hopf C. Nursing on Haemodialysis 1962-1966. Manoscritto non pubblicato, aprile 1996.

34. Fox RC, Swazey JP e Cameron EM. Social and ethical problems in the treatment of end-stage renal disease patients. In: Narins RG (ed). Controversies in nephrology and hypertension. New York: Churchill Livingstone, 1984, pp.45-70.

35. Albers J. Reflections on the Dialysis Nurse Training Program. ANNA Journal 1989; 16:230-231

36. Castagnoli A. L'assunzione obbligatoria al lavoro con riferimento ai nefropatici. In: Prevenzione e riabilitazione nell'insufficienza renale, Atti Convegno Nazionale AROER, Bologna 10-11 ottobre 1975, pp.295-296.

37. Dingwall RR. 25 Years of Caring Together. A short history of EDTNA/ERCA.1996, p.15.

38. Cinte J, Thon That $\mathrm{H}, \mathrm{Bec} \mathrm{Ph}$, et al. L'hémodialyse périodique dans le traitement de l'insuffisance rénale chronique. La Revue du Praticien 1971; 21: 2806-2810.

39. I dati si riferiscono ad una comunicazione non pubblicata, fornita dall'AOSTED - Associazione Operatori Sanitari in Tecniche Emodialitiche nel corso dei lavori del $\mathrm{I}^{\circ}$ Congresso Nazionale svoltosi a Tirrenia nel 1987. Nella terza parte si parlerà più diffusamente di tale associazione.

40. Van Waeleghem JP. The Changing Role of the Neprology Nurse. In: EDTNA Proceedings 1981; 9: 14-21.

41. Van Waeleghem JP, Gammar N, Lamberty MC, et al. Development of Nephrology Nursing Care in Europe: 19781988. ANNA J 1989; 16: 233-236. 